\title{
On the Use of Subwavelength Radial Grooves to Support Spoof Surface-Plasmon-Polariton Waves
}

\author{
Mario F. Pantoja, Zhi Hao Jiang, Pingjuan L. Werner, and Douglas H. Werner
}

\begin{abstract}
This letter introduces radial grooves as a feasible geometry to support spoof surface-plasmon-polariton (SPP) waves propagating along a vacuum-metallic interface. Through the analytical derivation of a dispersion relation, validated also with full-wave simulations, the ability of radial grooves to operate in the fundamental mode at the microwave regime is demonstrated. Also, results demonstrate the utility of radial geometries to decrease the depth of the grooves, in comparison with the widely used rectangular grooves, thereby providing a superior methodology for generating spoof SPPs.
\end{abstract}

Index Terms-Microwave propagation, plasmons.

\section{INTRODUCTION}

$\mathbf{R}$ ECENT advancements in the development of thin-film devices and low-dimensional materials have created a pathway towards mimicking the unique properties of plasmonics at microwave frequencies. In this sense, a seminal paper [1] proposed using rectangular grooves as structures which were able to partially reproduce the behavior of dielectric-conductor SPPs in the microwave regime. The idea behind this strategy is to create effective reactive surfaces supporting TE-polarized waves [2]. This represents a challenging problem due to the requirement that the conductor (i.e., metal) needs to exhibit a negative real part of the permittivity, which does not appear naturally at microwave frequencies. Consequently, they are named as "spoof" or "designer" SPPs. Moreover, the complementary approach reported in [3] emphasized the role of the waveguides for the analysis of the physical principles of operation, which in fact were suggested several years earlier in [4]. Since then, the search for new possibilities to exploit the similarities between grooved metal structures and the true SPPs has been an extremely active area of research [5], [6]. For example, graded rectangular [7], thin rectangular [8], and trapezoidal grooved structures [9], [10], among others, have been proposed. The desirable properties of spoof SPPs include: a) low-frequency operation, which can facilitate dispersion

Manuscript received May 21, 2016; accepted July 19, 2016. Date of publication October 21, 2016; date of current version November 4, 2016. This work was supported in part by the Spanish and Andalusian research programs TEC2013-48414-C3-01 and P12- TIC-1442. The authors would like to thank the Spanish Ministry of Education-Commission Fulbright Program "Salvador de Madariaga" (PRX14/00320) for sponsoring the joint research between authors.

M. F. Pantoja is with the Department of Electromagnetics, University of Granada, 18071 Granada, Spain (e-mail: mario@ugr.es).

Z. H. Jiang, P. L. Werner, and D. H. Werner are with the Electrical Engineering Department, The Pennsylvania State University, University Park, PA16802 USA (e-mail: zuj101@psu.edu; plw7@psu.edu; dhw@psu.edu).

Color versions of one or more of the figures in this paper are available online at http://ieeexplore.ieee.org.

Digital Object Identifier 10.1109/LMWC.2016.2614970 relation calculations; b) low propagation losses, which can be assessed with the short-open calibration technique [11]; c) high confinement of the fields, which is proportional to $\delta=\left(\mathrm{k}^{2}-\mathrm{k}_{0}^{2}\right)^{-1 / 2}$, with $k$ and $k_{0}$ the propagating and space momentum, respectively; d) low dispersion, related to the group velocity and derived also from the dispersion relation; e) preservation of the original boundary, which requires shallow grooves; and f) controllability of the former properties, which means that small variations of the groove dimensions can have a significant impact on the characteristics of the SPP waves.

This letter represents a step ahead in the analysis of microwave structures able to support spoof SPPs, by presenting the well-known family of radial grooves as ideally suited candidates for this purpose. In fact, the propagating modes of these structures are well-known in electromagnetics due to their long history in applications such as wedge waveguides [12] or annular-sector resonators [13]. In essence, what makes them attractive for application to the spoof SPP wave problem is the gradual cutoff of their propagating mode that requires the existence of a radial coordinate $\rho_{r}$, which separates spatially propagating and non-propagating sectors of the waveguide [12]. For the particular case of spoof SPPs, where it is desirable to have a high attenuation in short lengths of propagation so as to reduce the depth of the grooves, the employment of radial grooves improves the ability to mimic true SPPs. This issue has also been pointed out through full-wave simulations for trapezoidal grooves [9], [10]. Alternatively, this letter provides an analysis of the radial grooves by deriving a novel dispersion relation, which also has the advantage of decreasing the computational burden of the required simulations. Furthermore, the boundary conditions applied are expressed in terms of port impedances, which links the actual problem with the classical theoretical works based on waveguides [12], [13]. The results included also compare the performance of radial and rectangular grooves, thus confirming the relevance of the proposed structures to enhance the behavior of spoof SPPs.

\section{Theoretical Analysis and Dispersion Relation}

Fig. 1 depicts a subwavelength unit-cell based on the inclusion of radial grooves forming a one-dimensional (1D) array of waveguides along the surface of a perfect electric conductor (PEC). The dimension of the unit cell is $d_{u}$, while the aperture width, height and total angle of the radial grooves are $a, h$, and $\varphi_{g}$, respectively. To derive the dispersion relation for this structure, we follow the procedure outlined in [1], 


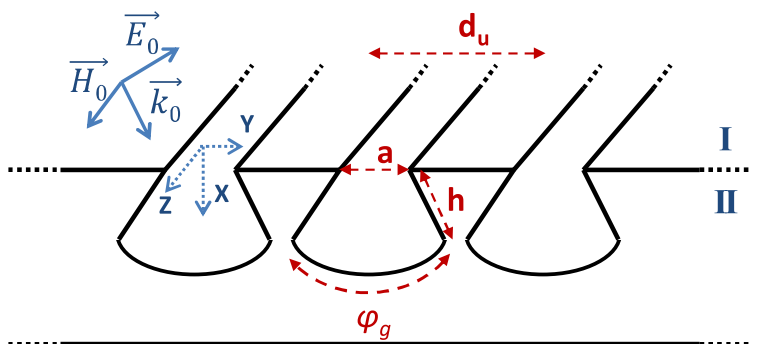

(a)
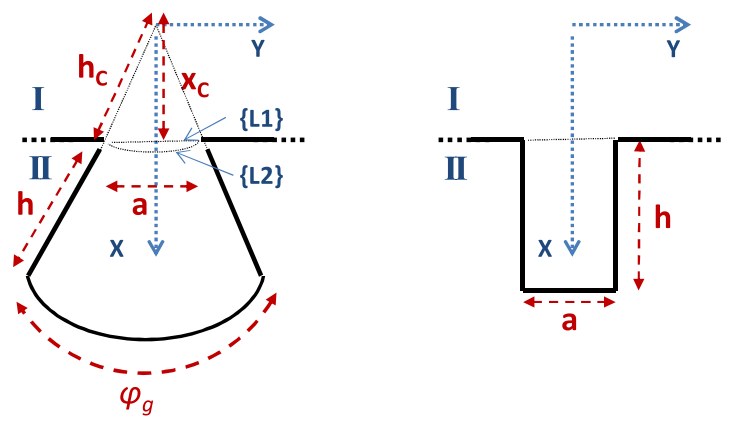

(b)

Fig. 1. (a) $1 \mathrm{D}$ array of radial waveguides, immersed in vacuum with intrinsic impedance $\eta_{0}$. (b) Dimensions and reference system for radial (left) and rectangular (right) grooves.

where a $p$-polarized incident plane wave $\left(\vec{E}^{i n c}, \vec{H}^{i n c}\right)$ with a wavenumber $k_{0}$ and parallel momenta $k_{x}, k_{y}$ is assumed to impinge on the perfect conducting boundary of the structure. Under these conditions, fields in region I can be expressed as

$$
\left\{\vec{E}^{I}, \vec{H}^{I}\right\} \approx\left\{\vec{E}^{i n c}, \vec{H}^{i n c}\right\}+r_{0}\left\{\vec{E}^{r e f, 0}, \vec{H}^{r e f, 0}\right\}
$$

where it is considered that the grooves operate at subwavelength frequencies, and the only contribution to the reflected fields $\left(\vec{E}^{r e f}, \vec{H}^{r e f}\right)$ results from the specular diffraction order $r_{0}$. Also, fields inside the grooves (region II) can be written as a linear combination of the forward and backward propagating fundamental modes such that

$$
\begin{aligned}
\vec{H}^{I I} & =C^{+} H_{0}^{(2)}\left(k_{0} \rho\right) \hat{z}-C^{-} H_{0}^{(1)}\left(k_{0} \rho\right) \hat{z} \\
\vec{E}^{I I} & =\hat{\varphi} \frac{j \eta_{0}}{k_{0}} \frac{\partial H_{z}^{I I}}{\partial \rho}=\hat{\varphi} j \eta_{0}\left[C^{-} H_{1}^{(1)}\left(k_{0} \rho\right)-C^{+} H_{1}^{(2)}\left(k_{0} \rho\right)\right]
\end{aligned}
$$

where $(\rho, \varphi)$ represent the cylindrical coordinates inside the groove (Fig. 1), and $H_{n}^{(j)}$ are $n$-th order Hankel functions of the $(j)$ kind, where $j=1$ or 2 .

Reflection coefficients can be derived by applying the standard boundary conditions. Thus, the tangential component of the electric field $E_{\phi}^{I I}$ must be zero at $\rho=h+h_{C}$, where $h_{C}=(a / 2) \operatorname{cosec}\left(\varphi_{g} / 2\right)$, which leads to

$$
C^{-} / C^{+}=H_{1}^{(2)}\left(k_{0}\left(h+h_{C}\right)\right) / H_{1}^{(1)}\left(k_{0}\left(h+h_{C}\right)\right) .
$$

Additionally, at the surface of the perfect conductor, the tangential component of the electric field must be continuous $\left(E_{y}^{I}=E_{y}^{I I}\right)$. The third and final boundary condition applies for the magnetic field at the same surface, $H_{z}^{I}-H_{z}^{I I}=j_{s z}$ where $\vec{j}_{s}$ is the current density on the PEC. In particular, the following boundary condition can be applied:

$$
\begin{aligned}
& \frac{\int_{L_{a}} E_{y}^{I} d y}{\hat{x} \times\left(H_{z}^{I}\left(a_{i}\right)-H_{z}^{I}\left(a_{f}\right)\right)} \\
& =\frac{\int_{L_{a}} E_{y}^{I I} d y}{\hat{x} \times\left(H_{z}^{I I}\left(a_{i}\right)-H_{z}^{I I}\left(a_{f}\right)\right)+j_{s z}\left(a_{i}\right)-j_{s z}\left(a_{f}\right)}
\end{aligned}
$$

where $L_{a}$ is the aperture of the groove at the surface, and $a_{i}$, $a_{f}$ are the initial and final points of this aperture, respectively. After some manipulations, (4) may be used to show that

$$
\begin{aligned}
- & \frac{j \eta_{0} k_{x}}{k_{y} k_{0}} \frac{1+r_{0}}{1-r_{0}} \\
= & \frac{j \eta_{0} \int_{x_{C}}^{\rho_{u}} \frac{d \rho}{\sqrt{\rho^{2}-x_{C}^{2}}}\left[H_{1}^{(2)}\left(s_{h}\right) H_{1}^{(1)}\left(k_{0} \rho\right)-H_{1}^{(1)}\left(s_{h}\right) H_{1}^{(2)}\left(k_{0} \rho\right)\right]}{\left[1-\sin \left(\varphi_{g} / 2\right)\right]\left[H_{1}^{(1)}\left(s_{h}\right) H_{0}^{(2)}\left(s_{C}\right)-H_{1}^{(2)}\left(s_{h}\right) H_{0}^{(1)}\left(s_{C}\right)\right]}
\end{aligned}
$$

where $\rho_{u}=\sqrt{(a / 2)^{2}+x_{C}^{2}}$, with $x_{C}=(a / 2) \sec \left(\varphi_{g} / 2\right)$, $s_{h}=k_{0}\left(h+h_{C}\right)$ and $s_{C}=k_{0} h_{C}$.

Taking into consideration the divergence properties of $r_{0}$ leads to the dispersion relation for the surface bound state, and considering a cutoff propagating mode in the groove:

$$
j \eta_{0} \frac{\sqrt{k_{y}^{2}-k_{0}^{2}}}{k_{y} k_{0}}=S_{0}\left(k_{0}\right)
$$

where the right-hand side of (5), given as $S_{0}\left(k_{0}\right)$, can be numerically calculated.

Alternatively to (5), an approximate analytical equation can be formulated through the identification of $S_{0}\left(k_{0}\right)$ in $(6)$ as the input impedance of an annular-sector waveguide [13]:

$$
Z_{\text {in }}\left(k_{0}\right)=-j \eta_{0} \frac{\varphi_{g} a}{d} \frac{J_{1}\left(s_{C}\right) Y_{1}\left(s_{h}\right)-Y_{1}\left(s_{C}\right) J_{1}\left(s_{h}\right)}{J_{0}\left(s_{C}\right) Y_{1}\left(s_{h}\right)-Y_{0}\left(s_{C}\right) J_{1}\left(s_{h}\right)}
$$

Differences among these formulations are expected in part because (7) was derived by applying radial apertures at the input port (which implies an integration path $\rho=h_{C}\{\mathrm{~L} 2\}$ as in [13] instead of the path $x=x_{C}\{\mathrm{~L} 1\}$ employed here and depicted in Fig. 1(b). Also, additional differences may arise because the quotient of the Poynting vector and the square of the electric field at the aperture were used to derive (7) rather than the ratio of electric/magnetic field applied in (5).

\section{RESULTS}

Figure 2 shows the validation of the dispersion relation by a comparison of the resonance frequency calculated using (5), (7) and full-wave simulations based on CST Microwave Studio ${ }^{\circledR}$. A set of examples covering different groove angles and heights were simulated, which correspond to cases where the grooves have the same volume. Roots of the non-linear dispersion relation were obtained numerically by a secant method. As mentioned above, it can be appreciated that for small inner angles, equations (5) and (7) provide similar results, yielding very good agreement with the fullwave simulations (deviation is on the order of $5 \%$ ). As the inner angle increases, the solution of (7) becomes more 


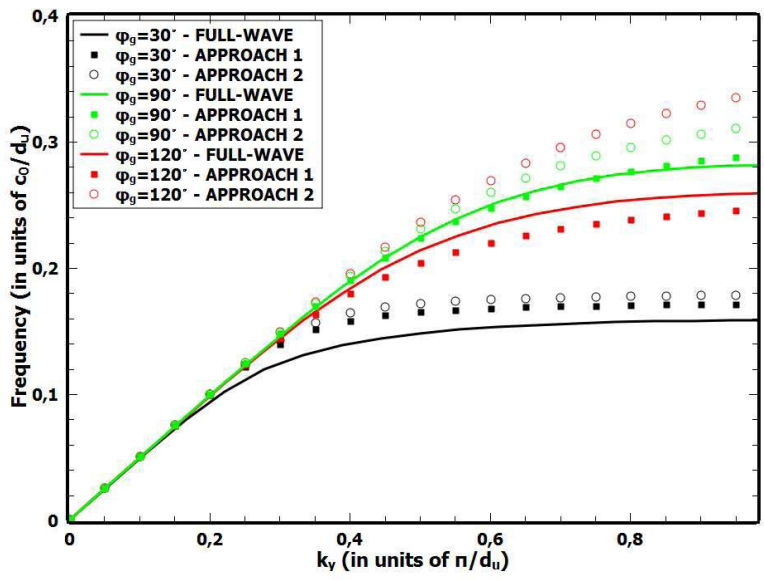

Fig. 2. Validation of the dispersion relation for radial grooves. Three cases are plotted, corresponding to: $\mathrm{h}=0.9294 \mathrm{~d}_{\mathrm{u}}$ and $\varphi_{\mathrm{g}}=30^{\circ}$ (black line), $\mathrm{h}=$ $0.2712 \mathrm{~d}_{\mathrm{u}}$ and $\varphi_{\mathrm{g}}=90^{\circ}$ (green line), $\mathrm{h}=0.1908 \mathrm{~d}_{\mathrm{u}}$ and $\varphi_{\mathrm{g}}=120^{\circ}$ (red line) The comparison includes: full-wave simulation (solid line), theoretical derivation of (5) (squares), and analytical approximation of (7) (circles).

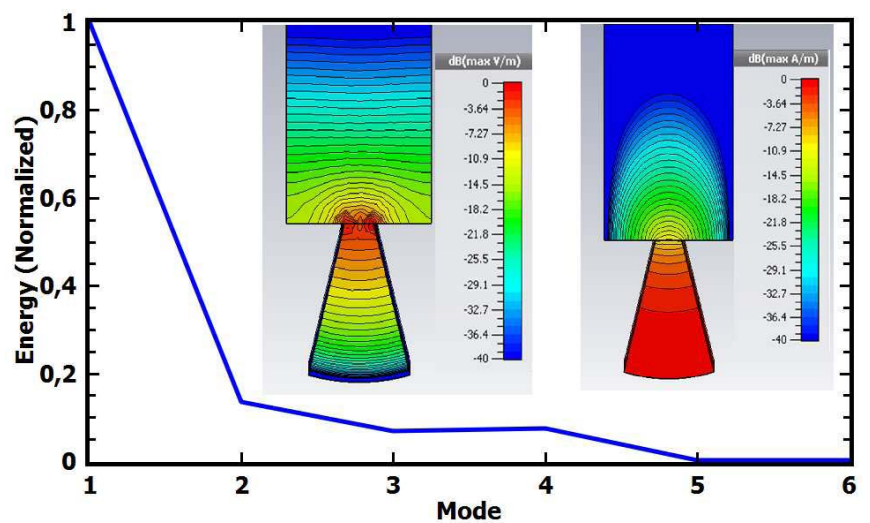

Fig. 3. Relative energy of the six lower propagating modes of the radial grooves. Inset shows the distribution of the tangential electric field (left) and normal magnetic field (right) at the fundamental mode, calculated using the full-wave solver CST Microwave Studio ${ }^{\circledR}$ (eigenmode solver).

inaccurate in comparison to (5), as initially expected because of the more exact integration path $\{\mathrm{L} 1\}$. Figure 3 illustrates the dominance of the fundamental mode, as well as the distribution of the electric and magnetic fields at the resonance frequency.

Finally, some analysis is carried out on the performance of the radial grooves. Fig. 2 confirms that the inner angle has a significant influence over the resonance frequency of the fundamental mode, thus suggesting that these structures are highly tunable. The main reason for this can be found in the abovementioned analogy with annular-sector waveguides, where the gradual cutoff mode represents a greater ability to attenuate waves when compared to rectangular waveguides [12]. To further explore this fact, Fig. 4 presents a comparison between rectangular and radial waveguides of the same height $h$ and aperture width $a$, clarifying the advantages in terms of miniaturization for the radial waveguides. A larger groove depth for the rectangular geometry (leading to similar volumes for both radial and rectangular grooves) equalizes the operating resonance frequency, but still presents a higher dispersion

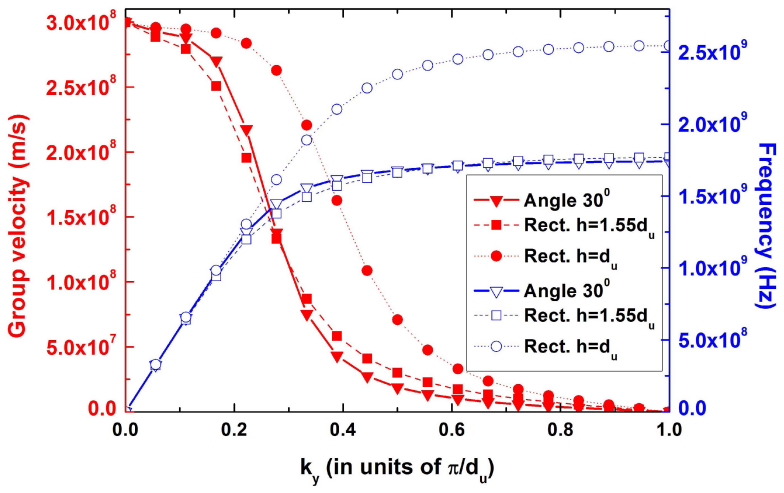

Fig. 4. Comparison of group velocity (left) and resonance frequency (right) of a structure with radial and rectangular grooves. A value of $a=0.2 d_{u}$ is used for the three cases considered. The radial grooves have an inner angle of $30^{\circ}$ with a depth of $\mathrm{h}=\mathrm{d}_{\mathrm{u}}$. A unit cell with $\mathrm{d}_{\mathrm{u}}=0.025 \mathrm{~m}$ was chosen.

in the group velocity (e.g., producing a higher phase delay) compared to the radial case. These advantages could eventually be exploited in wideband communication systems.

\section{CONCLUSION}

Radial grooves were shown to outperform rectangular grooves in their ability to sustain SPPs in terms of tunability. It was further demonstrated that this property can be used to decrease the vertical size of grooves as well as to simultaneously increase the operational bandwidth.

\section{REFERENCES}

[1] F. J. Garcia-Vidal, L. Martin-Moreno, and J. B. Pendry, "Surfaces with holes in them: New plasmonic metamaterials," J. Opt. A, Pure Appl. Opt., vol. 7, no. 2, pp. S97-S101, 2005.

[2] R. F. Harrington, Time-Harmonic Electromagnetic Fields. New York, NY, USA: McGraw-Hill, 1961.

[3] A. P. Hibbins, M. J. Lockyear, I. R. Hooper, and J. R. Sambles, "Waveguide arrays as plasmonic metamaterials: Transmission below cutoff," Phys. Rev. Lett., vol. 96, no. 7, pp. 073904-1-073904-4, Feb. 2006

[4] A. A. Maradudin and A. Sentenac, "The impedance boundary condition for a periodically corrugated metal surface," Solid State Commun., vol. 84, nos. 1-2, pp. 159-163, 1992.

[5] X. Shen, T. J. Cui, D. Martin-Cano, and F. J. Garcia-Vidal, "Conformal surface plasmons propagating on ultrathin and flexible films," in Proc. Nat. Acad. Sci. USA, vol. 110, no. 1, pp. 40-45, 2013.

[6] J. Y. Yin, J. Ren, H. C. Zhang, B. C. Pan, and T. J. Cui, "Broadband frequency-selective spoof surface plasmon polaritons on ultrathin metallic structure," Sci. Rep., vol. 5, pp. 8165-1-8165-5, Feb. 2015.

[7] Q. Gan and F. J. Bartoli, "Graded metallic gratings for ultrawideband surface wave trapping at THz frequencies," IEEE J. Sel. Topics Quantum Electron., vol. 17, no. 1, pp. 102-109, Jan./Feb. 2011.

[8] X. Wan and T. J. Cui, "Guiding spoof surface plasmon polaritons by infinitely thin grooved metal strip," $A I P A d v$., vol. 4, no. 4, pp. 047137-1-047137-8, 2014.

[9] F. M. Zhu, Y. Y. Zhang, L. F. Shen, and Z. Gao, "Subwavelength guiding of terahertz radiation by shallowly corrugated metal surfaces," J. Electromagn. Waves Appl., vol. 26, no. 1, pp. 120-129, 2012.

[10] X. Liu, L. Zhu, Q. Wu, and Y. Feng, "Highly-confined and low-loss spoof surface plasmon polaritons structure with periodic loading of trapezoidal grooves," AIP Adv., vol. 5, no. 7, pp. 077123-1-077123-7, 2015.

[11] H. Xiang, Q. Zhang, J. Chai, F.-F. Qin, J.-J. Xiao, and D. Han. (Mar. 2015). "Experimental measurements of fundamental and highorder spoof surface plasmon polariton modes on ultrathin metal strips." [Online]. Available: https://arxiv.org/abs/1503.00220

[12] S. F. Mahmoud, Electromagnetic Waveguides: Theory and Applications. U.K.: IET, 1991.

[13] G. B. Collins, Microwave Magnetrons. New York, NY, USA: McGraw-Hill, 1948, pp. 49-63. 